\title{
VIEJAS Y NUEVAS CUESTIONES EN LAS VIEJAS Y NUEVAS TERAPIAS PSICOLÓGICAS
}

\author{
Vicente PELECHANO \\ Facultad de Psicología, Universidad de La Laguna, Tenerife, Islas Canarias
}

\begin{abstract}
Resumen: Después de una toma de posición respecto a las terapias biológicas para los trastornos mentales, la psicología de la salud y la psicología positiva, se exponen ideas y resultados referentes a la eficacia de las terapias psicológicas tradicionales con la sugerencia de explicaciones alternativas a partir de las presencias y ausencias de aspectos relevantes en los estudios publicados. Se apuntan sugerencias a partir de los estudios de meta-análisis de las terapias psicológicas, así como algunas de las aportaciones de las terapias inspiradas en la psicología positiva. La delineación de unas conclusiones tentativas apoyan la necesidad por una mejor y más cualificada investigación sobre eficacia de terapias en la que se insista en la interacción entre terapeuta y cliente, así como las cualidades de cada uno de ellos, y en donde los procedimientos empleados sean menos relevantes y se acentúe la presencia de sucesos externos a las sesiones y curso de la terapia y que inciden definitivamente sobre ella.
\end{abstract}

Palabras clave: Valoración de terapias psicológicas, psicología clínica de la salud, psicología clínica positiva, terapias de la «nueva era».

Old and new topics in old and new psychological therapies

\begin{abstract}
After a reflection on biological therapies for mental disorders, health psychology and positive psychology, ideas and study results are presented as to the efficacy of traditional psychological therapies, offering alternative interpretations that take into account the presence or the absence of relevant aspects in published investigations. Suggestions are made based upon metaanalyses of psychological therapies, as well as on contributions derived from positive psychology. The outlines of tentative conclusions point to the need for more and better studies as to the efficacy of those therapies that hinge on therapist-client interaction and their characteristics, and where procedure is less relevant but the presence of events is underlined that are external to the sessions and the course of the therapy though do have a definite influence thereon. Some reflections on illustrative «new therapies» and the «new age therapies» are exposed.
\end{abstract}

Keywords: Efficacy and efficiency of psychological therapies, clinical health psychology, positive clinical psychology, therapies of a new era.

\section{INTRODUCCIÓN}

Hace unos años, la psicología clínica acaba de cumplir, oficialmente, su primer centenario de vida y representa una especialización psicológica indudable y una actividad profesional reconocida en prácticamente todos los países en los que existe psicología. Desde sus inicios ha

Recibido: 5 junio 2007; aceptado 12 junio 2007.

Correspondencia: Vicente Pelechano, Universidad de La Laguna, Facultad de Psicología, Tenerife, Islas Canarias. Correo-e: vpelecha@ull.es. existido una dualidad esencial entre los practicantes: aquéllos que han defendido que su actividad es una rama de la psicología y por ello deben aplicarse todos los recursos metodológicos, teóricos y procedimentales propios de la psicología como ciencia y, aquellos otros que o bien han intentado «crear» una ciencia al margen de la ciencia (como sucedió con el psicoa-

Agradecimientos: Este trabajo ha sido posible gracias al proyecto SEJ2006-14301/PSIC, Consolider, Bien-S-Star, sin que exista ningún compromiso entre los miembros del equipo y las tesis que aquí se defienden, que son de exclusiva responsabilidad del autor de este trabajo. 
nálisis y la fenomenología) o bien se han posicionado claramente frente a ella. En los últimos años, esta dualidad básica sigue estando presente y se encuentra vestida con colores que, parecen nuevos y como tales se están ofreciendo al consumidor (tanto psicólogos clínicos como pacientes). Si bien no toda la psicología clínica es reducible a las intervenciones terapéuticas (como toda medicina no se restringe a la cirugía), parece fuera de toda duda que el psicólogo clínico profesional echa mano de los recursos de intervención (las terapias) con el fin de aliviar los problemas que se le presentan. Y todavía existen fuertes discusiones acerca de qué modelo y teoría es mejor para el tratamiento, cuando en realidad teorías y modelos no siempre guardan una relación clara con los procedimientos que se aplican.

El número de terapias y/o procedimientos terapéuticos que se han inventariado pasan del millar y el hecho de que existan no quiere decir, sin más, que sean útiles ni eficaces por sí mismas; simplemente se utilizan sin saber, en muchos casos sobre qué se actúa y el efecto que poseen (a medio y largo plazo). En este sentido, ofrecer datos y reflexiones respecto a la bondad de las terapias no parece fuera de lugar (efectos, tanto positivos como negativos). Por otra parte, el balance final acerca del cuerpo doctrinal de la psicología clínica en general y de las terapias psicológicas en particular, así como su alcance se encuentra en entredicho, al menos, desde tres perspectivas: la consideración acerca de su eficacia, las denominadas «terapias de la nueva era» y algunas partes de la psicología clínica positiva. Nunca antes de ahora ha existido una demanda tan frecuente de servicios de asistencia «clínica» psicológica y nunca han proliferado tal cantidad de procedimientos asentados en intuiciones o «evidencias» subjetivas tan controvertidas como en los últimos veinte años. Y, junto a todo ello, aparecen aspectos críticos respecto a la práctica profesional, su cualificación y su valor hasta el punto que uno de los peligros que se vislumbran es el debilitamiento progresivo de las «terapias psicológicas» debidas a la mala aplicación, o aplicación indiscriminada y por lo mismo inadecuada, a los «clientes» que la demandan (una especie de muerte por el éxito, y si no muerte, al menos, estado muy debilitado y ciertamente enfermizo). Es verdad que la psicología clínica cubre más campos que los correspondientes a las terapias (entre ellos, la psicopatología, aunque también el asesoramiento y consuelo psicológico) $\mathrm{y}$, en la última década, los elementos que se van incorporando progresivamente de la «psicología positiva», pero también lo es que estas incorporaciones tienden a confundir realidad con deseo de éxito de los creadores, y moda con resultados contrastados.

Ha surgido, además, como una especialidad que está creciendo y consolidándose, la «psicología de la salud», que posee una versión «clínica» denominada en los últimos años tentativamente como «psicología clínica de la salud» y que se ocupa sustancialmente de los problemas psicológicos que presentan los enfermos «físicos», así como de cuestiones relacionadas con utilización, optimización y mejora de los servicios de salud y del funcionamiento psicológico de los distintos profesionales que trabajan en el mundo sanitario. La irrupción del psicólogo en el sistema de prestación de servicios de salud y no solamente de salud mental, muy posiblemente represente una nueva «revolución» en la prestación de servicios y su actuación ha sido, hasta el momento, positiva para mejorar estos servicios, aunque queda todavía mucho por hacer.

En este trabajo nos limitaremos a ofrecer datos y reflexiones sobre las terapias «psicológicas» y su eficacia, con dos anotaciones complementarias aunque esenciales: la psicología de la salud y la psicología positiva; y un aditamento previo sobre las terapias biológicas en psicología. La primera, en sus implicaciones y aplicaciones en la clínica; la segunda, asimismo en los intentos, todavía incipientes, para crear una alternativa a la psicología clínica convencional. Y el aditamento, porque nos encontramos en plena efervescencia y renovación de las terapias biológicas para problemas psicológicos como parte de un intento por refundar de nuevo a la misma psicología. Explícitamente, no se trata de una revisión, sino de una toma de posición respecto a un conjunto de temas que son de interés en las terapias psicológicas, con el propósito de que el lector vaya perfilando su propia posición (no necesariamente coinciden- 
te con la que aquí se expone), o la reafirme después de haber terminado con la lectura.

\section{BIOLOGÍA Y TERAPIAS BIOQUÍMICAS PARA LOS TRASTORNOS MENTALES}

Por lo que se sabe hasta nuestros días, todo conocimiento se encuentra mediado por estructuras corporales, aunque ese conocimiento no se limita a este compromiso biológico. Y toda actividad psicológica conocida trae consigo una contrapartida biológica en el sentido de que no tenemos conocimiento científico que no se encuentre sostenido por una estructura y una función biológica (sin cuerpo no existimos como seres humanos). Claro está que de estos datos no resulta científicamente lícito reducir toda acción psicológica a una acción biológica, de la misma manera que una acción biológica no se reduce a una acción física a partir del reconocimiento de la base física de los fenómenos biológicos. La tentación reduccionista (de uno u otro sentido) ha estado presente desde hace siglos e incluso puede ser considerada como acicate y estímulo de investigación (por la navaja de Occam de no multiplicar los entes sin necesidad) aunque debe tenerse muy claro que, al menos en los niveles de análisis, modelos y conocimientos que se manejan en nuestros días, este intento está condenado al fracaso (y se aceptan más las posiciones emergentistas tipo Holland que las meramente reduccionistas).

Es verdad que los avances en la investigación de la bioquímica y de los psicofármacos han representado una mejora indudable en muchos casos por lo que se refiere a los síntomas observables desde los lloros y la desesperación a las conductas agresivas. Pero, a la vez, existe una convicción cada vez más aceptada de que la acción bioquímica alivia la sintomatología pero no elimina el problema de la ansiedad, la depresión, la agresividad o la esquizofrenia por poner tres ejemplos conocidos o, por decirlo de otra manera, que la ansiedad, la depresión, la agresividad y la esquizofrenia son mucho más que los fármacos que se aplican y su acción bioquímica. Aparte, claro está, de los «efectos secundarios» o no deseados que presentan los fármacos, desde dependencias a efectos rebote de agravamiento de los problemas al dejar de consumirlos.

Y existen casos claros de fracaso: al margen del tipo de clasificación utilizada, los denominados trastornos de personalidad son problemas ante los que los fármacos poseen una acción muy limitada, en muchas ocasiones, nula y en alguna hasta paradójica, cuando no contraproducente. La depresión que aparece en los trastornos límite de personalidad no remite con ninguno de los antidepresivos conocidos $\mathrm{y}$, en más de una ocasión, éstos incrementan la probabilidad de suicidio. Y la falta de eficacia se anota, al menos en nuestros días, en la mayoría de los trastornos de personalidad inventariados, desde el dependiente al antisocial o disocial. Es posible que se defienda la idea de que los «trastornos de personalidad» no existen o que deberían ser eliminados de las distintas nosologías. Sin embargo, la eliminación del nombre no elimina los problemas; y los problemas son que no existen fármacos eficaces para la eliminación, siquiera, de una gran parte de la sintomatología que se presenta en este tipo de trastornos, se llamen de una o de otra manera.

Una de las ideas que va teniendo una aceptación cada vez mayor entre los profesionales es que la acción más importante de los fármacos es que, al aliviar una parte de los disparadores de los síntomas perturbadores (bloqueando la hiperactivación o hipoactivación), «posibilitan» la acción de los recursos psicológicos que todavía se encuentran presentes en las personas y, por ello, actúan como mejoría de ciertos problemas que serían solucionados por los propios pacientes o por las interacciones entre pacientes y otras personas significativas (entre ellas el o la terapeuta). Dentro de este marco general se apuntan ciertas interpretaciones de interacción entre fármacos y terapias psicológicas, que han sido un tipo de solución, no siempre con éxito, aunque muy recomendada en los últimos cuarenta años.

Posiblemente las limitaciones en la acción de los psicofármacos deban atribuirse a las notas definitorias del modelo biológico de los trastornos mentales. La detección de bases biológicas sigue siendo fiel al modelo categorial dicotómico de presencia o ausencia de una enfermedad dada si existen o no ciertos elementos (por 
ejemplo, el vacilo de Koch en el caso de la tuberculosis) y no se está más o menos tuberculoso por la presencia de más o menos bacilos. En ciencia, aparte de la «presencia» se exige en muchos casos, asimismo, una gradación en cantidad y esta exigencia es la que se aplica a la instrumentación y aplicación de la psicología y de sus terapias. Seguramente en algunos casos y problemas, el acercamiento dicotómico es más adecuado a la realidad que se estudia (como en las infecciones bacterianas y en los traumatismos) pero en otros, predomina una lógica cuantitativa (calibración) en la que además de detectar la presencia, se insiste en la evaluación de la cantidad (o su carencia) para poder explicar los fenómenos y atacarlos de manera más eficaz, y una consideración de una variedad de fenómenos que inciden en la conducta o enfermedad observable. Esta calibración no es práctica usual en el modelo biológico al uso, aunque se están haciendo intentos de aproximación, como la genética cuantitativa defendida por autores como Plomin, con la inclusión de modelos multivariable en el estudio de la determinación de los fenómenos. Aún así, la bioquímica, como tal, se encuentra muy distante, en nuestros días, de reflejar la complejidad psicológica que presenta la psicopatología y, desde ahí, las posibilidades de acción terapéutica. Y el anexo final de muchos tipos de discurso defendiendo la existencia de una base biológica, aunque «no conocida todavía», no resuelve ninguno de los problemas que existen.

Otro punto que posee cierto interés es el de la especificidad de los fenómenos estudiados y de los efectos que se observan. En el caso de las alteraciones, no existe una relación punto a punto entre prescripciones farmacológicas y problemas psicológicos (de hecho existen muchos más «modelos» que tipos de psicofármacos activos). En muchas ocasiones los problemas en el diseño del estudio llevan a conclusiones «científicas» que no son tan específicas como defienden los «resultados» alcanzados referidos a bases biológicas e incluso psicológicas de los problemas biológicos o psicológicos. Y en esta cuestión de la inespecificidad existen muchos ejemplos como los que se refieren a la personalidad tipo A, la etiología del maltrato infantil y el «reflejo biológico cerebral» de las madres de niños autistas. El tipo A se presentó como la esperanza preventiva y de intervención para el infarto de miocardio (una de las primeras causas de mortalidad en los países occidentales) en los años setenta del pasado siglo: veinte años después se conocían demasiados resultados incoherentes, cuando no contradictorios con estas predicciones y en la actualidad no se acepta que se trata de un patrón que se encuentre presente específicamente en los problemas cardiovasculares graves. De hecho, hemos encontrado importantes componentes del patrón A en ciertos cánceres y en enfermedades renales.

Se ha dicho en reiteradas ocasiones, que el maltratador infantil lo que hace es reproducir el maltrato que recibió en la infancia a partir de unos datos en los que, sobre muestras de maltratadores, un poco menos de la mitad de ellos aproximadamente, informaban haber recibido malos tratos de sus padres y nada se sabe acerca de la «otra mitad», ni de los que, habiendo recibido mal trato infantil no se han convertido en maltratadores cuando son mayores. De nuevo, la especificidad, brilla por su ausencia.

Finalmente, una de las últimas modas en psicología es la aplicación de la imagen computarizada cerebral como elemento diferenciador entre personas con ciertos diagnósticos graves o entre personas que interactúan con ellas (por ejemplo, las madres de niños autistas, frente a madres de niños «normales» presentan un patrón de activación cerebral distinto): el problema, claro está, es que nada se dice respecto a la especificidad del patrón frente a todo otro tipo de «madres» y de «niños», ni se argumenta un razonamiento causal al respecto (al menos, afortunadamente, por ahora) por lo que se puede estar concluyendo erróneamente lo que es inespecífico de ciertos problemas (como lidiar con autolesiones, agresividad y estereotipia de respuestas, todas ellas juntas o un tipo de problemas común a todos ellos) con lo que es específico de uno nada más. El problema de la especificidad de fenómenos y soluciones que sobre ellos se propongan no existe solamente en los modelos biológicos, aunque en ellos se encuentra un sesgo importante de discurso tanto para entender los fenómenos psicológicos, como para lograr su modificación. 


\section{LA PSICOLOGÍA DE LA SALUD Y LA CLÍNICA PSICOLÓGICA.}

En el último cuarto del siglo XX se ha observado una entrada decidida de la psicología en el mundo de la salud «física». Desde la consideración de la salud como un fenómeno que cubre más dominios que el biológico $\mathrm{y}$, entre ellos, el psicológico, la psicología ha mostrado su utilidad en el estudio de las enfermedades crónicas (desde las creencias sobre la enfermedad hasta la adherencia al tratamiento y la utilización de los servicios de salud). En el tratamiento de muchas enfermedades crónicas (el cáncer es un caso ilustrativo aunque de ninguna manera el único), en intervenciones quirúrgicas y su recuperación posterior, así como en secuelas de traumatismos creaneoencefálicos y demencias, la psicología ha comenzado a colaborar de manera eficaz y los problemas ante los que se ha encontrado no son todos reducibles a la psicología clínica convencional que se ocupaba solamente de los «trastornos mentales» identificados en las correspondientes clasificaciones.

Todo ello ha ofrecido un nuevo horizonte de definición en el que la original psicología clínica se está diversificando en tantas especialidades como «servicios médicos» (algo similar a lo que ha ocurrido con la medicina), y en cada caso, requiere especificaciones y concreciones de formación encaminadas a incrementar su eficacia. Además y muy especialmente, el mundo infantil y el de la tercera y cuarta edad son especialidades emergentes, con procedimientos terapéuticos específicos y dirigidos a estas poblaciones (y sus familias). Ello quiere decir que la primitiva psicología clínica se encuentra sobrepasada, en estos últimos años, por la demanda de modelos, técnicas y procedimientos que se apliquen a poblaciones «físicamente enfermas» con el fin de aliviar su sufrimiento y posibiliten que la familia pueda soportar la carga de tener un enfermo crónico entre sus miembros (en la actualidad, casi la mitad de la población adulta en los países occidentales avanzados), con lo que la dedicación a esta tarea es no solamente conveniente sino que exigirá la realización de una gigantesca tarea de investigación que, ya en muchos lugares, comienza a ser interprofesional y que, sin duda, sobrepasa la formación dada a los psicólogos clínicos hasta este momento ${ }^{1}$.

\section{LA TERAPIA EN LA PSICOLOGÍA CLÍNICA POSITIVA}

Nos encontramos en plena «década de la conducta» tal y como fue preconizada por Seligman a finales del siglo XX. A partir del «sesgo» negativo que se encontraba presente en la mayor parte de libros de psicología «serios»" se ha propuesto equilibrar la situación a base de estudiar variables que como la felicidad, el bienestar psicológico, el optimismo, el perdón o la sabiduría, deberían formar parte explícitamente de la formación del psicólogo. Aunque la pretensión de «novedad» es más una cuestión de mercado que de realidad, la psicología «positiva» ha dado ya frutos con instrumentación, modelos e incluso terapias asentadas en los resultados teóricos y empíricos obtenidos hasta la fecha. Dos ejemplos de ello se encuentran en la propuesta de «crecimiento personal» tras experiencias traumáticas de Tedeschi y Calhoun $(1996,2004)$ y la «terapia del bienestar» de Ruini y Fava (2004).

La idea de «crecimiento post-traumático» después de sufrir un trauma es muy antigua (aprender de los fracasos) y se encuentra presente en la mayor parte de modelos sobre el ser humano, el descubrimiento de significado (Coger, Kemeny, Taylor y Fahey, 1998), así como en el reconocimiento de emociones positivas, incluso después, o en situaciones muy estresantes (Folkman y Moskowitz, 2000). Se arranca del hecho de que en algunas personas, el haber sobrevivido a una experiencia traumática les lleva a un replanteamiento de vida, buscan más la propia intimidad e incluso pueden aparecer reconversiones espirituales acompañadas de filosofías de vida más satisfactorias que

\footnotetext{
${ }^{1}$ Una reflexión sobre todo ello. La mayor parte de bibliografía publicada en la psicología de la salud se centra en algunas enfermedades: cáncer, infarto cardiovascular, sida y en todo caso, diabetes, cubren casi todo el campo. Y quedan muchas más alteraciones y enfermedades apenas tratadas hasta el momento. No quiere esto decir que lo que se ha hecho no sea relevante, sino que lo que queda lo es tanto o más.
} 
las que tenían con anterioridad. No quiere esto decir que se promueva la aparición de traumas para lograr un cambio «positivo» en la vida sino que en estas circunstancias, todavía no bien conocidas, es posible apelar, después de cierto tiempo, a replanteamientos emocionales y cognitivos profundos en la vida de las personas y que estos replanteamientos no necesariamente les lleven a una situación de sufrimiento generalizado sino que, en muchos casos, es posible reconstruir su vida a base de ayudar a replantear un esquema de vida del superviviente, saber atender y analizar los problemas sin una necesidad casi compulsiva para resolver de inmediato los problemas, insistir en el proceso de recuperación más que en el trauma pasado y animar a generar un nuevo esquema cognitivo y emocional.

En la denominada «terapia del bienestar»se parte de la idea de que con las terapias tradicionales sobre depresión y ansiedad, queda un remanente de recaídas y molestias (Ramana et al., 1995) que se dejan sin tratar en la medida en que los clientes ya se incorporan a su vida diaria. A partir del esquema teórico propuesto por Ryff (Ryff y Singer, 1996) se trabajan las seis dimensiones del bienestar psicológico: (1) dominio del contexto de vida; (2) crecimiento personal; (3) propósito y/o sentido de la vida; (4) autonomía en establecimiento de expectativas y valoraciones; (5) aceptación personal; y (6) relaciones positivas con los demás. Existe un programa de ocho sesiones (una semanal o cada dos semanas) con una duración entre 30 y 50 minutos cada una de ellas. Se han llevado a cabo investigaciones controladas sobre fases residuales de trastornos afectivos (Fava, Rafanelli, Cazzaro et al., 1998), prevención de depresión recurrente (Fava, Rafanelli, Grande, Conti y Belluardo, 1998), pérdida de efecto clínico o rebote de síntomas depresivos durante el tratamiento (Baldesarini, Ghaemi y Viguera, 2002) y ansiedad generalizada (Ruini y Fava, 2002). En todos ellos se ha comparado esta terapia del bienestar con procedimientos convencionales bien sea de fármacos o de acercamientos comportamental-cognitivos más convencionales, y los resultados han sido positivos.

La aportación, hasta ahora, de esta psicología positiva en la terapia es más bien escasa, con resultados también escasos y, en todo caso, como aspectos que pueden ser relevantes aunque ciertamente «secundarios». Conceptualmente, en las terapias psicológicas se han estado utilizando elementos incentivadores positivos de manera sistemática desde los inicios de la terapia de conducta (la aplicación de recompensas y el entrenamiento asertivo son dos ejemplos ilustrativos) y, además, con una unidad de análisis, posiblemente, más precisa y concreta. Al menos en lo que se ha ofrecido hasta el momento con intentos sistematizadores (véanse, p. ej., los volúmenes compilados por Aspinwall y Staudinger, 2003; Linley y Joseph, 2004; Lopez y Snyder, 2003; y Snyder y Lopez, 2005) de bastantes páginas, son, de hecho, más sugerenciales que sistemáticos, incitadores más que solucionadores de problemas, y en ellos abundan redundancias conceptuales que no se detectan y ni siquiera son denunciadas por los editores de los volúmenes (véase, por ejemplo, el capítulo sobre esperanza de Lopez et al. 2004 y la carencia de raíces históricas y teóricas). En suma, parece que se trata de un acercamiento que se encuentra en un estadio de desarrollo más bien escaso, muy activo, aunque con niveles exigitivos científicos bastante dispares entre sí, ante el que hay que permanecer atento y a la vez, exigir tratamientos científicos mas precisos; la propuesta de Seligman (1993) acerca de profundidad de los fenómenos psicológicos involucrados en el proceso y sensibilidad al cambio no pasa de ser un anuncio que deja fuera más cosas de las que toma en cuenta (la apelación a dificultad y generalidad de fenómenos y atributos está siendo eje central de experimentación y teoría en el modelo de parámetros desde 1973 hasta nuestros días, con más de una docena de tesis doctorales, varias monografías y más de medio centenar de artículos).

\section{QUÉ ES UNA TERAPIA PSICOLÓGICA}

Antes de entrar en la valoración de las «terapias psicológicas» convendría fijar su significación, lo que no resulta ni simple, ni fácil. Hacia mitad del siglo pasado, Raymi (citado en Eysenck, 1961) llegó a definir la psicoterapia como «una técnica no identificada, aplicada a 
problemas inespecíficos, con resultados impredecibles. Para ello recomendamos un entrenamiento riguroso», que jocosamente, se refería a la considerable variedad de procedimientos, estilo, maneras, lugares e incluso profesiones que se dedicaban a ella (desde entonces las profesiones y procedimientos han crecido más de un $400 \%$ ). Unas décadas después, en la obra de referencia compilada por Bergin y Garfield (1994) ni siquiera se intentó una definición y, en todo caso, se diferenciaba a la terapia del consuelo psicológico por formación de los encargados de ponerlas en práctica, estructura y objetivos a alcanzar. Sin que se haya llegado a un consenso académico respecto a modelos y procedimientos, en la tercera edición del libro de Frank (Persuasión y curación, 1991) se recoge una delimitación que es insatisfactoria por lo que se refiere a su estado académico pero es descriptiva de muchas de las notas que la identifican. Una terapia psicológica se realiza (a) en un lugar, usualmente el despacho del terapeuta, pensado para recibir ayuda necesaria por un sufrimiento emocional y que fortalecería las expectativas de recibir ayuda por parte del paciente; (b) establece una relación entre paciente y terapeuta denominada «relación terapéutica» con papeles bien diferenciados para ambas partes; (c) el terapeuta posee un modelo teórico que utiliza para explicar el sufrimiento que presenta el paciente (modelo preferiblemente científico o con inspiración científica); y (d) se aplican procedimientos terapéuticos que se derivan más o menos directamente del modelo que posee el terapeuta y en estos procedimientos participan activamente uno y otro $^{2}$.

\footnotetext{
2 Estas «notas» son orientativas y pese a su generalidad dejan fuera opciones tales como la clínica comunitaria en la que el «lugar» de tratamiento es, preferiblemente en el que se presenta el problema (familia, centros escolares, centros de trabajo); los modelos de «búsqueda» más que de «espera; la asignación o no de tareas para llevar a cabo fuera de las sesiones; los «tratamientos de grupo en centros escolares, etc. (Pelechano, 1979, 1980). Asimismo debería tenerse en cuenta la participación de para-profesionales en el tratamiento y que en ciertos problemas han resultado muy eficaces y deben hacernos recordar que existen elementos «terapéuticos» (al igual que patogénicos) fuera de las sesiones terapéuticas en sentido estricto y que desempeñan un papel muy activo tanto en la mejora como en el empeoramiento de los pacientes. Más adelante se hará una referencia a esto en otro contexto.
}

Alternativamente y de manera asimismo genérica se ha identificado a la terapia psicológica como la aplicación de los conocimientos de la psicología para aliviar el sufrimiento humano y/o fomentar el bienestar personal en situaciones y contextos en los que existe, prioritariamente, una necesidad o demanda clínica (si esta demanda no existe puede ser un problema a resolver por la psicología educativa o la social) y en la que participan dos o más personas (una de ellas, el terapeuta). Repárese que se trata de la psicología (toda la psicología, de entrada) y no solamente de una parte de ella y que no se delimita el modelo ni los procedimientos aunque sí se exigiría que los conocimientos a aplicar proviniesen de la psicología científica, lo que lleva consigo ciertos problemas de delimitación operativa y conceptual.

\section{EL PROBLEMA DE LA VALORACIÓN \\ DE LAS TERAPIAS PSICOLÓGICAS: UN TÓPICO RECURRENTE Y COMPLEJO}

Muy posiblemente tenga un escaso rechazo la afirmación de que las terapias psicológicas representan en la actualidad una actividad que ocupa a muchos profesionales, posee una considerable demanda social (tanto en la prestación de servicios como en la demanda de formación por parte de psicólogos y de alumnos de últimos cursos de graduación) y, por todo ello, representa un considerable volumen de movimiento de capital, así como una gran inversión de recursos humanos. Cabría pensar, desde aquí, que debe existir un interés considerable en el conocimiento de sus procesos, determinantes y eficacia, así como de su implementación. El caso es que aunque representa un foco de interés, no es central ni en el mundo académico ni en el profesional, y tampoco parece que sea un foco de atracción para los investigadores. Hace ya 30 años dedicamos al tema una publicación (Pelechano, 1977) que ha visto varias ediciones (p. ej., Pelechano y Botella, 1983) y en la actualidad, aunque ha habido algunos cambios, sustancialmente siguen existiendo problemas similares a los que allí se 
detectaron, con algunos intentos de solución (meritorios aunque no han ofrecido solución alguna) y, a la vez, con algunas amenazas. No existe un único procedimiento que responda a la cuestión y, los tipos de soluciones propuestas han sido las siguientes:

(a) Un primer recurso para conocer la eficacia de una u otra terapia es el estudio de caso. Originalmente es el más antiguo y, posiblemente el primero de todos los procedimientos a utilizar. La ventaja que posee este procedimiento es que se tienden a exponer (sobre todo en los primeros momentos en los que se está gestando la terapia) con detalle y claridad los supuestos, modos operativos y resultados obtenidos. Entre sus desventajas se encuentran que solamente se publican los éxitos terapéuticos, que no existe control acerca de las múltiples interacciones y momentos que se dan en las sesiones, que la generalización a otros casos es más que problemática y poco dice aparte de ofrecer ideas para los profesionales acerca de lo que está actuando. Además, no existen controles adecuados ni información acerca de los acontecimientos externos que suceden mientras se están realizando las sesiones. Sirven a los profesionales con ideas y sugerencias y muestran con claridad que existe una separación entre procedimientos concretos y modelos teóricos que inspiran las terapias. De ahí la necesidad de emplear otros recursos.

(b) Esencialmente el estudio acerca de la validación de las terapias psicológicas arrancó en 1952 con el trabajo pionero de H. J. Eysenck, revisado después en 1961 y 1966 por este autor, y por Rachman en 1971. Planteado en términos de ciencia natural, la aportación de Eysenck fue duramente criticada por el análisis elemental de exposición de resultados (porcentajes de mejora y/o de curación) aunque ningún autor le acusó de que los resultados presentados fueran falsos o estuvieran equivocados. Sustancialmente, el trabajo de Eysenck estuvo dedicado a estudiar la eficacia de la terapia psicoanalítica y de las terapias eclécticas, a lo que añadió Rachman la terapia de Rogers, así como el tratamiento con niños y con psicóticos. El resultado general en todas las revisiones fue claro: por lo que se refiere a lo que se denominaban «neurosis», tres cuartas partes de los pacientes en los estudios realizados, mejoraban a los cuatro años sin terapia alguna y, en el caso de las terapias psicodinámicas, los resultados de mejora en este mismo período no pasaba del $66 \%$, por lo que la conclusión parecía obvia: las terapias psicodinámicas, en el mejor de los casos, perjudicarían la recuperación (la «remisión espontánea») de estos pacientes. En el resto de grupos diagnósticos, los resultados eran peores. La polémica estaba servida, así como la aparición de un nuevo campo de trabajo: el estudio de la eficacia diferencial de las distintas terapias. El planteamiento del tema coincidió con la irrupción de las terapias de conducta y, desde aquí, la conversión de estas nuevas terapias como la posible solución de todos los problemas.

Desde una perspectiva de ciencia naturalista, ya fue defendido por Eysenck el empleo del método experimental, que implicaría la formulación de hipótesis concretas derivadas de los distintos modelos terapéuticos, selección de los pacientes siguiendo procedimientos objetivos, asignación aleatoria de pacientes a distintos grupos de tratamientos (doble ciego de pacientes y terapeutas) y un grupo de control, al menos; y valoración antes y después de los tratamientos con el empleo de criterios y de instrumentación objetiva. En los últimos tiempos se suele añadir el control de covariables que pueden incidir en la eficacia de los distintos tratamientos sin que sean motivo concreto de intervención ${ }^{3}$. Para bien o para mal, no existen demasiados trabajos publicados en los que se haya utilizado un diseño experimental multivariable y los resultados hayan sido analizados dentro de esta lógica, con inclusión de covariados y de interacciones a lo largo del proceso. Posiblemente esta

\footnotetext{
${ }^{3}$ La verdad es que el uso de covariables es muy limitado pese a la importancia que poseen con el fin de poder atribuir los cambios a los procedimientos terapéuticos y no a otros procesos no estudiados. Cuando hemos llevado a cabo controles de este tipo los efectos debidos al tratamiento aparecen, aislados y con mayor claridad, aunque el trabajo se complica considerablemente, tanto en el diseño como en el análisis de datos y en los procedimientos estadísticos a emplear y estilo de discurso en la discusión de los resultados.
} 
ausencia ayuda a que las conclusiones que se han entresacado de los mejores trabajos publicados no hayan sido aceptadas por toda la comunidad científica.

Se han llevado a cabo muchos estudios que pueden asimilarse a trabajos experimentales controlados. Uno de los citados como referencia es el Estudio de Temple (Sloane, Staples, Cristal, Yorkston y Whipple, 1975) en el que participaron 94 pacientes ambulatorios, la mayoría con el diagnóstico de neuróticos y el resto con diversos trastornos de personalidad. Los pacientes fueron seleccionados de forma equilibrada respecto a género y gravedad de síntomas y asignados aleatoriamente a uno de dos grupos de tratamiento (psicoanalistas expertos y terapeutas de conducta expertos) y un grupo de control (espera de cuatro meses para recibir tratamiento y si tenían crisis, podían acudir a quien les realizó la entrevista de recepción). Todos los terapeutas tenían una gran experiencia en el tratamiento de casos, eran considerados como buenos profesionales y gozaban de una buena reputación como terapeutas. Antes de llevar a cabo las terapias se consensuaron líneas generales para contrastar las modalidades terapéuticas y a ellas se adhirieron los terapeutas. Se tomaron distintas medidas antes de comenzar el tratamiento y a los cuatro meses (se supone el final del mismo), con seguimiento al año y a los dos años de la evaluación inicial de las sesiones y se realizaron una media de 14 sesiones; asimismo se realizaron calificaciones sobre los pacientes por parte de un observador externo que no había participado en las terapias. En cuanto a resultados, tanto los pacientes tratados con terapia de conducta como los tratados con terapias psicoanalíticas presentaron una mejora en sintomatología (tanto en los instrumentos como en las calificaciones del observador externo). Realmente todos los pacientes mejoraron (incluso los del grupo de espera), aunque los tratados mejoraron más. Y estas mejorías se mantuvieron durante el período de seguimiento.

Sin embargo, este estudio no posee un valor definitivo y ha sido criticado desde distintos frentes. En primer lugar, los resultados hablan de las versiones «cortas» de las terapias y que se ocupan de los que se identificaban como pacientes neuróticos; en segundo lugar, se trata de «mejoría» pero no de curación ni eliminación de sintomatología con incorporación definitiva al mundo social (durante ese período la mejoría puede haberse producido por las acciones terapéuticas, pero asimismo, por motivación reactiva, generación de expectativa de mejora o tasa basal de cambio); en tercer lugar, se trata de «los mejores terapeutas posibles» con líneas generales de acción, pero no protocolos específicos de tratamiento, con lo que los contagios entre modelos y procedimientos no fueron controlados (existen procedimientos terapéuticos similares en los dos acercamientos, por ejemplo, el de enfrentamiento a la situación ansiógena); en cuarto lugar, los efectos a corto, medio y largo plazo se restringieron a unas parcelas concretas, con ausencia de estudios sobre generalización.

Y críticas desde un punto de vista científico se encuentran en otros casos y perspectivas. Un trabajo que se ha propuesto como ejemplo de realización es el Programa de Colaboración de Investigación para el Tratamiento de la Depresión del NIMH (Elkin, 1994). Comparó la eficacia relativa de dos acercamientos psicoterapéuticos (terapia cognitiva y terapia interpersonal), con una medicación antidepresiva (la imipramina, Tofranil). Al final, tanto las psicoterapias como la terapia farmacológica lograron resultados significativos y equiparables en comparación con un grupo placebo. Además, después de un seguimiento de 18 meses, no se encontraron diferencias significativas entre los grupos de tratamiento y, de los pacientes que habían mejorado inicialmente, el $30 \%$ o menos estaban sin síntomas depresivos.

La polémica surgió en los resultados respecto a la eficacia aparente de los fármacos frente a la psicoterapia: los investigadores que han analizado los mismos datos han deducido conclusiones distintas. Así, Klein (1996) concluyó que la imipramina tuvo efectos más beneficiosos en pacientes con depresión más grave, mientras que Jacobson y Hollon (1996) señalaron que los pacientes tratados con imipramina abandonaron con mayor frecuencia el tratamiento y presentaron más recaídas $\mathrm{y}$, por otra 
parte, estos últimos autores señalaron que todos los trabajos publicados han encontrado una superioridad de la terapia cognitiva frente a los fármacos en el tratamiento de la depresión. A todo ello hay que añadir la carencia de información respecto a las interacciones multivariable que se presentan en el análisis de casos reales y complejos como son las depresiones. Posiblemente los pacientes son mucho más que su «depresión» tal y como ésta es definida en los trabajos y en las taxonomías al uso, y la toma en consideración de variables de identificación y de funcionamiento psicológico que pueden influir en uno u otro momento del proceso terapéutico (tanto de personalidad como externas a la situación terapéutica como tal, véase más adelante) son relevantes y no han sido tomadas en consideración, entre otras cosas, posiblemente por la dificultad metodológica que ello trae consigo y la complejidad de análisis que exige.

El estudio comparativo de la eficacia de distintas terapias con estudios «experimentales controlados» posee como ventaja el intento por controlar variables que se han considerado relevantes para la eficacia terapéutica en condiciones controladas, si bien los diseños, a medida que son más nítidos y «controlados» se alejan de la complejidad de las situaciones y dinámica terapéutica y, además, todos ellos se encuentran aquejados de una concepción simplista y linear de efectos de las variables controladas. De ahí que, siendo ilustrativos del «método experimental», adolecen de serios problemas que les lleva a no poder ofrecer más que conclusiones parciales, detección de la relevancia relativa de alguna variable y mostrar si un acercamiento es igual, mejor o peor que otro en cuanto a resultados en las condiciones en las que se realiza el estudio, que no se asemejan mucho a las que se dan en la vida real fuera de los contextos de «laboratorio»y asimilados. Incluso los criterios de cambio, usualmente informes de terceros o cuestionarios, deben poseer unas características que no siempre se cumplen (por ejemplo, si son sensibles al cambio, los procedimientos de medida acerca de la fiabilidad se encuentran puestos en entredicho $\mathrm{y}$, además, no todos los procedimientos dan lugar a los mismos resultados).
La lógica discursiva del análisis experimental controlado ha ido evolucionando en las últimas décadas en un intento por aislar los componentes relevantes de las terapias. Las técnicas del desmantelamiento de los paquetes terapéuticos, así como el incremento en los estudios de seguimiento son ejemplos de ello. Y los resultados alcanzados demuestran sustancialmente dos cosas: en primer lugar, que con el seguimiento se tiende a perder parte de las ganancias $\mathrm{y}$, en segundo, que el desmantelamiento de componentes ha ofrecido una imagen deprimente en el sentido de que cada uno de ellos no es suficiente para la obtención de los resultados (recuérdese lo que sucedió a propósito de la desensibilización sistemática y lo que está sucediendo ahora en las «nuevas aportaciones» en el tratamiento de la ansiedad).

(c) Una tercera manera de abordar la eficacia de las terapias pretende acercarse a la puesta en práctica «real» de las mismas, tal y como éstas se hacen en las consultas de los profesionales. Estos estudios carecen del rigor metodológico que exigiría una respuesta científicamente adecuada a los problemas que se han presentado, pero recoge la percepción de la realidad terapéutica de los participantes (en especial, de los terapeutas y de los pacientes). En general, cuando se ha preguntado a pacientes y terapeutas sobre la mejoría obtenida como consecuencia de la terapia los resultados siempre han sido similares: los terapeutas consideran que los pacientes ha mejorado más que lo que afirman los propios pacientes.

Seligman $(1995,1998)$ utilizó Consumer Reports (una publicación estadounidense dedicada a valorar servicios y productos que consume el ciudadano y que posee 180.000 suscriptores), para lanzar una encuesta sobre satisfacción, por parte de los usuarios, de las terapias psicológicas recibidas. Los resultados alcanzados fueron sorprendentes: cerca del $90 \%$ de las personas que habían recibido ayuda por problemas emocionales reconocían que habían mejorado y la mejoría era más clara si habían recibido ayuda por parte de terapeutas cualificados y la duración de la terapia había sido, al menos, de seis meses. 
Es verdad que se trata de un estudio metodológicamente muy débil, con ausencia de controles (ausencia de datos sobre gravedad, entidad nosológica, remisión espontánea, etc.), aunque recoge una realidad social innegable en la que el paciente elige terapeuta porque cree que lo necesita, la duración del tratamiento no está fijada por el diseño, las técnicas a emplear se van adaptando a cada caso concreto, existen otros problemas además del «objeto prioritario de tratamiento» (comorbilidad) y los resultados implican tanto la eliminación de síntomas como indicadores de funcionamiento general. En todo caso, con sus imperfecciones y defectos, parece una realidad relacionada con la aceptación social y «buen nombre» de las terapias psicológicas para el tratamiento de los problemas emocionales, aunque poco o nada se sabe respecto a qué terapias son más eficaces, qué variables y sobre que personas son eficaces unos $\mathrm{u}$ otros procedimientos y de qué manera se actúa en ellas.

(d) Una cuarta opción ha sido y sigue siendo muy utilizada en la bibliografía y sobre todos los temas psicológicos. Es lo que podría calificarse como estudios narrativos, de los que los trabajos ya mencionados de Eysenck y Rachman son antológicos: se trata de ofrecer un panorama del estado de la cuestión a partir de una revisión, realizada por uno o más autores, de los trabajos publicados. El Annual Review of Psychology está compuesto básicamente por trabajos de estas características. Una de las críticas que se han formulado a este modo de hacer es que las percepciones, sistema de valores y creencias de los «narradores» influyen considerablemente en la selección, acento y relevancia atribuida a los trabajos que se incluyen (así como a los que se excluyen).

En un intento de superar estas limitaciones, Smith y Glass (1977) ofrecieron lo que se ha denominado desde entonces el meta-análisis en el que entrarían a formar parte los estudios que ofrecieran resultados, al menos, de un grupo control y otro experimental (tratado) y en el que el estadístico básico sería el tamaño del efecto (diferencia entre la media del grupo tratado y el grupo de control, dividido por la desviación típica del grupo de control), que es realmente una diferencia tipificada de medias, lo que permitiría la comparación ${ }^{4}$. Se trata de la consagración de la validación inter pares (en la decisión acerca de lo que entraría en el análisis: lo publicado en revistas con valoradores anónimos), no se entra directamente en el análisis de las condiciones metodológicas y en nada se toma en cuenta la bondad de los diseños y de los instrumentos, ni los criterios de diagnóstico ni de valoración de participantes. Smith et al. (1980) revisaron 475 estudios de resultados de psicoterapias y calcularon 1766 tamaños de los efectos, lo que comprendía, de hecho, todos o la mayor parte al menos, de los estudios publicados en los que era posible llevar a cabo una comparación. Los principales resultados pueden resumirse así: (i) El tamaño del efecto promedio fue de 0,85 , lo que implicaría más del 80 $\%$ de mejora; si se eliminan los tratamientos placebo y las estrategias de consolación el efecto sube a 0,93 ; (ii) Terapias de orientaciones teóricas distintas (psicodinámica, fenomenológica o científica) dan resultados equiparables. Asimismo ofrecen resultados similares las distintas modalidades terapéuticas (verbales, comportamentales, expresivas); las terapias más eficaces son las que se dirigen al tratamiento de la depresión, de las fobias simples y problemas similares; (iii) El tamaño del efecto tiende a ser independiente de la duración de las terapias, de si éstas se aplican de modo individual o en grupo y del grado de preparación y experiencia del terapeuta; (iv) El efecto de las terapias parece ir

\footnotetext{
${ }^{4}$ Claro que una cosa es que se permita la comparación y otra que esa comparación tenga sentido. Si los instrumentos utilizados son heterogéneos y algunos de ellos no cumplen con los criterios psicométricos (entre ellos corrección del error típico de medida), la comparabilidad entre resultados con instrumentos distintos y con distintas cualidades, más que estudio de «efectos» objetivos se acerca peligrosamente a una suerte de cuanticofrenia. Y argumentos del mismo porte deben emplearse a la hora de la aplicación del procedimiento a grupos de pacientes que se suponen «homogéneos» en función del diagnóstico utilizado (unas pocas características y sin control de fiabilidad ni de distintos tipos de validez). Este tipo de consideraciones ha sido esgrimido por críticos del meta-análisis desde posicionamientos científicos serios (bastante más serios que los argumentados por los defensores del procedimiento de valoración). Como cuestión fáctica, pese a todo lo dicho, en nuestros días se acepta el meta-análisis como un recurso más dentro de las posibilidades de análisis dentro de la psicología científica
} 
desapareciendo con el seguimiento: el tamaño del efecto, a los dos años, cae hasta un $0,50 \mathrm{y}$ un $9 \%$ de los casos, específicamente empeoran.

Trabajos posteriores con esta metodología han dado resultados, en parte, solapables con los del grupo de Smith (por ejemplo, el de Wampold, 2001). El resultado sobre la inespecificidad de los efectos de las distintas terapias se ha corroborado en un meta-análisis de 32 meta-análisis (Grissom, 1996). Respecto al «rigor» de la investigación, parece que posee un escaso efecto sobre el tamaño del efecto (Smith y Glass, 1977) ${ }^{5}$.

Existen muchos problemas con este procedimiento de valoración: realmente se trata de una estimación «de un solo nivel» en el que entra todo aquello que se publica, para bien y para mal. Nada se habla de la precisión diagnóstica ni de las variables tanto internas tomadas en consideración para el análisis, como «externas» que pueden haber influido en los cambios producidos en los pacientes, ni efectos motivacionales promovidos por la participación en unas sesiones en las que se les presta atención y que, pasado cierto tiempo, las «soluciones» encontradas en las terapias dejan de tener efecto (y nada se dice respecto a estas «soluciones», ni de los procesos involucrados en su puesta en acción). Finalmente, la existencia de «sesgos»" que parecían debilitar los estudios narrativos no han desaparecido en la medida en que los propios evaluadores «sesgan», seleccionan y criban aquellos aspectos que consideran relevantes y dejan de lado otros que pueden ser valiosos pero que no son tomados en consideración (la valoración inter pares no lleva consigo, por ella misma, una objetividad y, curiosamente, nada se dice respecto a los sesgos,

\footnotetext{
${ }^{5}$ Lo cual no es muy sorprendente en la medida en que la elección de instrumentación para la evaluación de los cambios y sus cualidades psicométricas no ha sido cuestión de análisis. La exigencia del diseño tiende a ir pareja con el rigor (no resulta esperable que se utilicen instrumentos muy exigentes en diseños y terapias que apenas lo son) aunque el «efecto» final de las diferencias puede ser similar. Esto es: la lectura del resultado puede ser distinta y caminar por la senda de que instrumentos distintos, con distinta calidad, son utilizados por autores distintos en distintos niveles de rigor de diseño y en el caso de agrupar los resultados, el estadístico empleado no es suficientemente potente para diferenciar entre los distintos modos de actuación.
}

defectos y errores en la valoración de los trabajos y, por otra parte, deberían ser «pares» objetivos, conocedores de los problemas y sin interés en uno u otro tipo de resultados... lo que no parece ser el caso; no existe una criba respecto a «buenos» y «malos» evaluadores de manuscritos a publicar). Esencialmente, además, no está claro si realmente con este procedimiento se valora lo que sucede, lo que deseamos que suceda o lo que «debe estar sucediendo». El meta-análisis, siendo meritorio, no pasa de ser un recurso sociológico, con las ventajas y desventajas que estos tipos de recursos llevan consigo.

(e) Ante el estado de variabilidad e incompletud de resultados como los presentados han aparecido dos opciones. La primera, a partir de los resultados de eficacia comparable entre distintas opciones terapéuticas y grado de formación y experiencia de los terapeutas ha estado defendiendo una preparación mínima de los terapeutas ( $\sin$ necesidad de doctorado ni graduación profesional especializada), como lo ha hecho Dawes (1966) y que defienden la incorporación de paraprofesionales a la práctica terapéutica. La segunda, desde dentro de la psicología académica, ha propuesto los «tratamientos empíricamente validados» como criterio de aplicación.

Por lo que se refiere a la participación de paraprofesionales, la evidencia empírica demuestra que son eficaces para el tratamiento de ciertos problemas relacionados con miedos y fobias infantiles, problemas de pareja y problemas familiares (véase Pelechano, 1979, 1980). Es un reto en la sociedad contemporánea ofrecer recursos de ingeniería comportamental que permita aliviar problemas que son o recurrentes o que se potencian con el estilo de vida actual. Y el mundo de la salud y las enfermedades crónicas y la participación de los familiares en el mantenimiento de la adherencia al tratamiento, así como en la colaboración en procesos de rehabilitación. Sin embargo, la participación de estos paraprofesionales no es una solución eficaz para todo tipo de problemas. Sabemos que se requiere una formación previa (que no se da en los sistemas educativos oficiales), y que a esta instrucción no se responde por 
igual por parte de todos los participantes, de manera que a veces se perturba más que se mejora con la participación de ciertos paraprofesionales que, llevados de buenas intenciones, llegan a romper todo un plan de intervención que podía ser muy eficaz; la familia puede ser parte de una solución, pero, asimismo puede ser, a veces, parte del problema y sin llegar a la propuesta psicoanalítica de que las madres de niños asmáticos eran generadoras o disparadoras de ataques, la experiencia clínica y la observación demuestran que existen familias que perturban considerablemente el bienestar de muchos de sus miembros y, por ello, la participación de la familia en programas de intervención debería estar precedida por una evaluación previa acerca de la disponibilidad de sus miembros, la preparación adecuada y las habilidades mínimas que se requieren para llevar adelante una ayuda eficaz.

La segunda opción a la que nos acabamos de referir se ha denominado las «terapias empíricamente validadas».. Un grupo de trabajo de la división 12 de la American Psychological Association (American Psychological Association Task Force, 1995) ha propuesto unos criterios de selección encaminados a aislar las terapias que cumplan a la vez con criterios científicos y de eficacia para los pacientes. Se diferenciaron dos grupos de tratamientos: los que denominaron como «bien establecidos» empíricamente (estudios con al menos dos grupos, realizados por investigadores distintos o, alternativamente, un gran número de estudios de caso con utilización de diseños experimentales, todos ellos realizados siguiendo manuales explícitos de aplicación y con una especificación clara de las características de los clientes) y, los calificados como «probablemente eficaces» (dos estudios en los que el tratamiento se muestra más eficaz que un grupo de control o al menos dos estudios bien diseñados en los que se demuestre eficacia al margen de la heterogeneidad de las muestras de pacientes, o estudios de caso bien diseñados). Esta opción, que ha tenido como modelo los criterios de la Federal Drug Administration para la aceptación de tratamientos en medicina, ha sido defendida por algunos autores (Nathan y Gorman, 1998) incluso como prescriptiva aunque asimismo ha sido fuerte- mente criticada (la «lista» se convierte, de hecho, en algo difícilmente modificable o en muy difícil de modificar, con lo que se dificulta la obtención de mejores procedimientos), además, algunas terapias, en la medida en que son formas de tratamiento asentadas en la buena fe llegan a alcanzar cotas de eficacia equivalentes a otras más estudiadas y establecidas (lo que resulta científicamente insatisfactorio aunque empíricamente correcto). Finalmente, los criterios de validación tienden a ser hechos respecto a las categorías del DSM (en la versión que corresponda), lo que representa la imposición de una taxonomía de consenso que no casa, con frecuencia, con las exigencias científicas que se utilizan para la validación terapéutica (y menos todavía sobre exigencias ideales de estudio científico).

(f) A partir de la separación entre investigación científica y práctica terapéutica se gestaron las que se han llamado «terapias de la nueva era» básicamente a partir de los años sesenta del pasado siglo en Estados Unidos (Singer y Nievod, 2003), como paso de una «nueva era astrológica» (de piscis a acuario) que traería consigo cambios personales, de prácticas profesionales y sociales y cambios políticos. Esta idea fue fermentada por la decidida entrada de religiones orientales (que fueron convenientemente modificadas para adaptarlas a la sociedad occidental) y creencias en las que se trataba no de rehabilitar al individuo con problemas (como pretendían las «viejas terapias») sino de transformarlo con el fin de que fuese uno con la naturaleza y el mundo que le rodea (tanto físico como espiritual). Así han surgido multitud de prácticas asentadas en la recuperación de memorias del pasado (que incluiría hipnosis, drogas de la verdad, fantasías guiadas, religiones basadas en oraciones y afirmaciones de que los problemas actuales están causados por uno o más traumas «olvidados» de otras vidas), la terapias de ritos satánicos y de entidades demoníacas, terapias del «re-nacimiento» y de la «repaternización», así como la terapia asentada en la existencia de una abducción previa por parte de extraterrestres. Se trata de un conglomerado de procedimientos alejados de la ciencia, que niegan la contrastación como criterio de 
validación y que suponen en la mayoría de los casos una conversión religiosa (acompañada de la pérdida de ahorros, del trabajo que se estaba desarrollando e incluso de la familia) y de un estilo de vida más bien marginal.

Estas «terapias de la nueva era» protestan cuando se valoran con procedimientos tradicionales tales como los expuestos más arriba. En la medida en que se establece una relación contractual que le cuesta dinero al paciente, estas terapias (y las antiguas también) están siendo «valoradas» mediante las intervenciones de las asociaciones profesionales y los tribunales de justicia y es en estos casos, cuando estas prácticas salen a la luz pública, con el subsiguiente escándalo y alarma social. Una cualificación profesional adecuada por parte de los clínicos que practican terapias, así como una vigilancia de asociaciones profesionales cualificadas y de la práctica profesional son recursos que cabe sugerir aunque nos encontremos, todavía lejos de poder ofrecer, en España, una red de controles que disminuya estas prácticas, que van creciendo, sin control, en los últimos años.

\section{UN INTERMEDIO NECESARIO: EL PROBLEMA DE LOS EFECTOS «NEGATIVOS»}

En la medida en que las terapias psicológicas persiguen el logro de un cambio y que resultan eficaces para lograr cambios, parece sensato pensar que los cambios que se logren pueden ser positivos o negativos. Sin embargo el análisis de los resultados «negativos» lleva consigo problemas específicos y no siempre bien delimitados. Un primer punto a considerar es la aparición de cambios producidos por la terapia o por el curso del problema que se está intentado paliar (el cambio puede estar producido por la evolución natural del trastorno más que por la intervención, lo que podría identificarse como el efecto resfriado: con antibióticos ocho días, sin antibióticos, una semana); en segundo lugar, la «extensión» de los cambios producidos (se puede dirigir la intervención hacia el logro de un objetivo concreto, como la eliminación de la fobia a cucarachas o a sectores fun- cionales más amplios); en tercer lugar, la «significación» de los cambios (estadística o clínica); en cuarto lugar, la consolidación de los cambios en el panorama general de evolución psicológica (si los cambios producidos se integran o son modificados con la evolución); en cuarto lugar los distintos tipos de «efectos negativos» tanto en procesos como en dominios funcionales. Al menos, se han considerado como efectos negativos a lo largo de la historia: (1) el abandono de las sesiones; (2) la ausencia de cambios significativos, aunque con mejoras no significativas; (3) ausencia de cambios significativos aunque con detección de deterioros no significativos y (4) deterioros significativos.

Falta por publicar un estudio sistemático de los efectos «negativos» de los tratamientos psicológicos. De entrada cabría pensar que si los tratamientos son poderosos para el logro del cambio, deben existir, como sucede en cirugía, errores y fallos profesionales (aparte, claro está, que se apliquen, a veces, procedimientos de manera inadecuada, procedimientos inadecuados o que no sean eficaces para el problema que presenta la persona). Los resultados publicados son mucho más optimistas: a mitad de los noventa (Stricker, 1995) se estimaba el efecto negativo entre $1 / 10$ y $1 / 13$ casos, aunque sin especificación de estos efectos. Cuando se trata de deterioro clínicamente significativo, Jacobson y Truax (1991) de una muestra de 30 pacientes que participaban en una terapia de pareja, solamente uno empeoró después de la terapia. En el proyecto de psicoterapia Vanderbilt II ninguno de los 61 pacientes que terminaron el programa presentó deterioro (Bein et al., 2000) y Ogles, Lambert y Sawyer (1995) informaron que en el estudio de colaboración sobre la depresión del NIMH solamente 1 de 250 pacientes presentó un empeoramiento significativo. Desgraciadamente, no existen datos duros publicados al respecto, por lo que se asume que las terapias psicológicas, en el peor de los casos, «no hacen daño» y en la práctica habitual mejoran a los pacientes. Este supuesto no se encuentra apoyado ni por modelos ni por resultados, entre otras cosas porque resulta difícil la publicación de resultados negativos. Caso de obtenerlos, tiende a atribuirse a defectos de procedimiento de aplicación, o de habi- 
lidad de los terapeutas...y no se suele publicar. Alguna monografía publicada y restringida a algunos procedimientos concretos de terapia de conducta tiene más de testimonio y elaboración teórica que de estudios sistemáticos. Y este estado de hechos resulta altamente insatisfactorio.

\section{EL INTENTO PARAMÉTRICO Y OMNICOMPRENSIVO DE MICHAEL LAMBERT (1992)}

En lugar de comenzar con un análisis empírico de resultados, Lambert llevó a cabo un extenso análisis racional de los trabajos publicados durante décadas en el campo y propuso un modelo de cuatro tipos de variables que incidían en la eficacia de las psicoterapias e incluso sugirió el porcentaje de éxito que debería atribuirse a cada uno de estos tipos. En este sentido han complementado la propuesta de Lambert, con inclusión de datos empíricos Hubble et al. (1999), Hubble y Miller (2004) y Miller et al. (1997). Se diferencian cuatro tipos de factores:

(1) Factores extraterapéuticos y del cliente. Son los que identifican al paciente, forman parte de las circunstancias de vida del paciente y de su recuperación al margen de la participación formal en la terapia. Consisten en las fortalezas que posee el cliente, elementos de apoyo con los que cuenta en su entorno e incluso elementos imprevistos y de azar. Ejemplos de tales elementos incluyen la persistencia, fe, un familiar de apoyo (marido, mujer, abuela, etc.), sentido de responsabilidad personal, membrecía de un movimiento religioso, logro de un nuevo trabajo o una crisis que ha sido vencida con éxito. La propuesta de Lambert es que este tipo de factores explicaba el $40 \%$ del éxito de las terapias; el reanálisis de Hubble y Miller (2004) a partir de datos procedentes de metaanálisis (Wampold, 2001) eleva el porcentaje al $87 \%$, más del doble. Este cambio se explica por la inclusión, en el proceso terapéutico de los elementos que el paciente cree que funcionan en su caso (que incluyen creencias preexisten- tes sobre el cambio, metas, tareas de la terapia y la alianza terapéutica o manera de introducir todos estos elementos dentro del proceso de recuperación), lo que implica los «factores comunes» (véase apartado siguiente sobre factores relacionales).

(2) Factores relacionales. En el modelo de Lambert se les atribuye un $30 \%$ de la varianza de resultados terapéuticos y se refieren a lo que en la bibliografía se denominan «factores comunes»: se trata de un conjunto de variables que definen la relación terapéutica y que son de la orientación terapéutica: cuidado, empatía, calor humano, aceptación, afirmación mutua, alianza terapéutica y ánimo para la aceptación del cambio y el riesgo del cambio son algunos de estos factores relevantes. La mayor parte de la varianza se incluye en el primer apartado de la refundición de Hubble y Miller.

(3) Placebo, esperanza y expectativa. Se refiere a la parte del cambio derivada del conocimiento de los pacientes de ser tratados y la evaluación de la credibilidad de la lógica expuesta y seguida por el terapeuta. Lambert propone que su aportación al cambio es del $15 \%$. En gran medida, formarían parte de los elementos comunes cuyo análisis entraría en el primer apartado y que no depende de ninguna técnica propuesta por modelos terapéuticos concretos.

(4) Factores dependientes del modelo y de la técnica concreta aplicada. Pueden ser interpretados como rituales específicos o rituales sanadores siguiendo la terminología de Frank (Frank y Frank, 1991): incluyen una manera de ver la realidad, una explicación sobre las dificultades que presenta el paciente y una variedad de estrategias y procedimientos que deben aplicarse para resolver estos problemas. En la propuesta de Lambert este tipo de factores explicaría el $15 \%$; en la revisión y propuesta de Hubbler y Miller (2004) la cifra baja hasta un $8 \%$.

La conclusión, en este caso, parece bastante clara. Si, en el mejor de los casos, el éxito terapéutico atribuible a técnicas y procedimientos específicos (que formarían la mayor parte del tiempo y contenidos de formación de los terapeutas) va de un 8 a un $13 \%$, parecería excesi- 
vo y sin un gran apoyo empírico, seguir promoviendo un cultivo de técnicas de cambio en lugar de un conocimiento de variables del sujeto y extraterapéuticas. Tanto la formación como la dinámica de las terapias psicológicas, o cambian radicalmente, o se encuentran en una situación de impasse de difícil salida.

\section{UNOS EJEMPLOS «NOVEDOSOS» Y SOMETIDOS A ESCRUTINIO}

Posiblemente estas consideraciones son las que explican que el cultivo de «nuevas terapias» siga siendo una vía fructífera para los buscadores de novedades que se encuentran escasamente apoyadas en los conocimientos psicológicos que tenemos. Ejemplos de estas novedades son el reprocesamiento y desensibilización del movimiento ocular (Eye Movement Desensitization and Reprocessing, EMDR de Shapiro, 1989), la terapia del campo de pensamiento (Thought Field Therapy, TFT, de Callahan, 1994) y las técnicas calificadas por Loftus y Kecham (1994) como «recuperación de memoria».

La técnica EMDR de reprocesamiento y desensibilización del movimiento ocular, posiblemente es el tratamiento nuevo con mayor difusión en Estados Unidos, con varios libros dedicados a su exposición (tanto de divulgación como de formación de técnicos). Han sido entrenados por encima de 30.000 psicólogos desde su presentación, con un instituto que se dedica a entrenamiento en dos niveles y una sociedad internacional (incluso ha sido objeto de distintas emisiones de televisión, entre las que se cuenta un noticiario en la cadena $\mathrm{ABC}$ ). Se ha utilizado para trastornos de ansiedad y experiencias traumáticas. El procedimiento pide que el paciente construya y mantenga una representación imaginativa de la situación traumática (o ansiógena) y que rememore las sensaciones físicas. Coincidiendo con ello, el terapeuta induce un conjunto de movimientos oculares de lado a lado, pidiendo que el paciente siga el movimiento de un dedo. Se pide al paciente que manifieste las cogniciones negativas que acompañan el sufrimiento emocional y que genere una aproximación más positiva sobre el trauma y lo que el paciente experimenta de él. La base teórica aducida es una especie de sistema inmune psicológico, a base de un procesamiento acelerado de la información a lo que ayuda el procedimiento de movimiento ocular (por el cambio de un procesamiento disfuncional a otro funcional). Las revisiones publicadas acerca de su base teórica y eficacia, sin embargo, dejan bastante que desear: (a) existe una escasa o nula relación entre la teoría expuesta por Shapiro y los datos que conocemos sobre ansiedad y psicopatología; y (b) los resultados utilizando técnicas de desmantelamiento de tratamiento elimina cualquier valor de elementos específicos no contemplados en otros acercamientos comportamental-cognitivos (el propio Shapiro llega a admitir que el movimiento ocular puede ser sustituido por otro, sin justificación alguna al respecto).

Los resultados conocidos respecto al TFT de Callahan (una mixtura entre fisiología teórica y acupuntura) son peores: no existe más que un trabajo publicado en una revista y en él, no existen controles de tratamiento convencionales, pese a su diseminación «cultural» entre terapeutas en Estados Unidos (Lohr, Hooke, Gist y Tolin, 2003).

En cuanto a las técnicas de recuperación de memoria, que incluyen hipnosis y diversos procedimientos de regresión, existe un considerable cuerpo de resultados demostrativos de que no deberían utilizarse como «recuperación» sino más bien como «implantación» de recuerdos y existe más de una recomendación de asociaciones profesionales en Estados Unidos que prohíbe que formen parte del repertorio profesional de los terapeutas (Lynn, Lock, Loftus, Krackow y Lilienfeld, 2003). Ni siquiera se reconoce como elemento de prueba en muchos tribunales de Estados Unidos.

En definitiva: el terapeuta debe estar abierto a novedades científicas, pero ello no implica «estar a la moda», o que se confunde lo último y quizás más impactante siguiendo los medios de comunicación con lo asentado científicamente, que es lo que debería guiar sus pasos. 


\section{COMENTARIOS ¿FINALES? Y SUGERENCIAS}

Después de más de un siglo de quehacer científico y profesional, las terapias psicológicas aparecen como un conglomerado de modelos y procedimientos que, aunque no resuelven todos los problemas para los que les piden ayuda, representan un volumen de recursos, muchos de los cuales han mostrado su eficacia ante una considerable cantidad de problemas, si bien la mayoría de ellas habría que ubicarlas en la categoría de mejoría o alivio, sin que se hayan resuelto de forma satisfactoria los problemas tratados. En los casos más frecuentes y aplicados (ansiedad y depresión), se logran alivios pero, en muchos casos, aparecen recaídas que no han encontrado ni una solución, ni una explicación satisfactoria. No existe una solución definitiva ante este estado de hechos y las «nuevas» aportaciones terapéuticas apenas han avanzado (incluso es dudoso que haya habido avances) en las últimas tres décadas. Ante esta situación se proponen las siguientes líneas de trabajo:

(1) En el estado actual de conocimiento y desarrollo de la psicología existe una escisión progresivamente mayor entre conocimientos psicológicos y aplicaciones terapéuticas. La pretendida escisión entre ciencia básica y ciencia aplicada parece corresponder más bien a la existencia de una ciencia de calidad y otra que no lo es tanto; de la primera, pronto o tarde se encuentran aplicaciones de peso (como sucedió con el conductismo skinneriano del análisis experimental de la conducta) que, aunque no definitivas, representan un avance claro en maneras de pensar y hacer. De la segunda, tras un primer impacto muy intenso (en el que muchos se ocupan de esta manera de ver), como la moda, tiende a desaparecer sin dejar más que unos cuantos científicos que siguen publicando sobre ello y no hay aplicaciones profesionales sino un cierto regusto de «modelo testimonial» (la indefensión representa un ejemplo, aunque no es el único, como el patrón tipo A). Aunque resulta difícil diferenciar, de entrada, ambas aportaciones, sería necesario hacer un proyecto, incluso un tanto aventurado, para la formación de los psicólogos clínicos que recogiera lo sustantivo de los modelos y aportaciones serias de la psicología, así como de la metodología, y encaminado a ofrecer conocimientos y actitudes ante los problemas psicológicos que respetara su complejidad y la multiplicidad de codeterminantes, así como se promoviera el estudio de la evolución de los problemas psicológicos a lo largo del ciclo vital y con la intervención de las interacciones que se dan en el mundo social. Y ello implicaría prescindir de un gran volumen de publicaciones y resultados que no son solamente redundantes, sino, en gran manera, irrelevantes y que sirven para llenar páginas de publicaciones sin que representen un avance en el conocimiento científico.

Ello representa un cambio en la filosofía de base, estructura y contenidos sobre la formación de los psicólogos clínicos. Y, junto a esta depuración en la formación tradicional, deberían incorporarse contenidos respecto a las enfermedades físicas y sus condicionamientos psicosociales, con el fin de que se conocieran los fundamentos básicos para poder intervenir en enfermedades crónicas (a lo largo de las distintas fases por las que se pasa) y en situaciones en crisis. Incluso esta última consideración representa un cambio sustancial respecto al conocimiento de las bases biológicas de la conducta.

(2) Incorporar de manera decidida las variables del individuo a los estudios, tanto en la realización, como en la valoración de las terapias. El modelo terapéutico al uso se asienta sobre una concepción de enfermedad física tradicional en la que se trata de identificar los patógenos (o focos disfuncionales) y actuar sobre ellos, prescindiendo del organismo vivo (individuo) sobre el que estos patógenos actúan. Esta incorporación del individuo exige tanto la identificación de las debilidades como de las fortalezas del individuo que recibe la asistencia psicológica. Y este planteamiento puede llevar a modificaciones sustantivas en la terapia en la medida en que no se tratan problemas en abstracto sino problemas que presentan individuos en los contextos de vida. 
Este posicionamiento exige un acercamiento individual a los problemas sin renunciar a las exigencias de la metodología científica. Sería más un posicionamiento idiotético que idiográfico. En este sentido, las sugerencias procedimentales de Lamiel pueden considerarse como un punto de partida.

(3) El quehacer terapéutico «controlado» se realiza en las sesiones terapéuticas, aunque para cada paciente, su vida no se restringe, de ninguna manera, a estas sesiones. El terapeuta debería tener información e incorporar a su trabajo terapéutico, los sucesos relevantes de la vida del paciente. Muchos estados de ansiedad y depresión, promovidos o mantenidos por la pareja, la inestabilidad económica y los conflictos en interacción personal, han mejorado y pueden mejorar mucho si la pareja vuelve a estar cariñosa con el paciente, tiene el paciente un golpe de fortuna económico, algunos de los miembros de su familia encuentran trabajo o arreglan los problemas que tienen con sus parejas... sin que el terapeuta tenga responsabilidad alguna en todo ello. Sin información tanto de los sucesos positivos como de los negativos relevantes y de su papel en la terapia, los resultados conocidos no pueden ser evaluados objetivamente, para asignar las responsabilidades correspondientes a los cambios promovidos por los procedimientos terapéuticos y por estos cambios «externos».

Es muy posible que estas reflexiones no resuelvan todos los problemas que presentan en la actualidad las terapias psicológicas, aunque pueden ayudar, y mucho, a que los estudios que se realicen siguiendo estos criterios arrojen luz acerca de lo que sucede con ellas. Y, desde aquí, avanzar en su validación real y en su mejora.

\section{REFERENCIAS}

American Psychological Association Task Force (1995). Template for developing guidelines: Interventions for mental disorders and psychological aspects of physical disorders. Washington DC: Autor.

Aspinwall, L.G., \& Staudinger, U.M. (Eds.) (2003). A psychology of human strengths. Fundamental ques- tions and future directions for a positive psychology. Washington, DC: American Psychological Association.

Baldesarini, R.J., Ghaemi, S.N., \& Viguera, A.C. (2002). Tolerance in antidepressant treatment. Psychotherapy and Psychosomatics, 71, 177-179.

Beinb, E, Anderson, T., Strupp, H.H., Henry, W.P., Schacht, T.E., Binder, J.L., \& Butler, S.F. (2000). The effects of training in time-limited dynamic psychotherapy: Changes in therapeutic outcomes. Psychotherapy Research, 10, 119-131.

Bergin, A.E., \& Garfield, S. (1994). Handbook of psychotherapy and behavior change ( $4^{\text {th }}$ ed.). New York: Wiley.

Dawes, R.M. (1994). House of cards: psychology and psychotherapy built on myth. New York: Free Press.

Elkin, I. (1994). The NIMH Treatment of Depression Collaborative Research Program: Where we began and where we are. En A.E. Gerbin \& S.1. Garfield (Eds.), Handbook of psychotherapy and behavior change $\left(4^{\text {th }}\right.$ ed.). New York: Wiley.

Eysenck, H.J. (1952). The effects of psychotherapy: An evaluation. Journal of Consulting Psychology, 16, 319324.

Eysenck, H.J. (1961).The effects of psychotherapy. En H.J. Eysenck (Ed.), Handbook of abnormal psychology. New York: Basic Books.

Eysenck, H.J. (1966). The effects of psychotherapy. New York: International Science Press.

Fava, G.A., Rafanelli, C., Cazzaro, M., Conti, S., \& Grandi, S. (1998). Well-being therapy: A novel psychotherapeutic approach to residual symptoms of affective disorders. Psychological Medicine, 28, 475-480.

Fava, G.A., Rafanelli, C., Grandi, S., Conti, S., \& Belluardo, P. (1998). Prevention of recurrent depression with cognitive behavioural therapy. Archives of General Psychiatry, 55, 816-820.

Frank, J.D., \& Frank, J.B. (1991). Persuasion and healing: a comparative study of psychotherapy ( $3^{\text {rd }}$ ed.). Baltimore: Johns Hopkins University Press.

Grissom, R.J.(1996). The magical number .7 + -.2: Metaanalysis of the probability of superior outcome in comparisons involving therapy, placebo, and control. Journal of Consulting and Clinical Psychology, 64, 973-982.

Hubble, M.A., Duncan, B.L., \& Miller, S.D. (1999). Directing attention to what Works, En M.A. Hubble, B.L. Duncan \& S.D. Miller (Eds.), The heart and soul of change: What works in therapy. Washington DC: American Psychological Association.

Hubble, M.A., y Miller, S.D. (2004). The client: psychotherapy's missing link for promoting a positive psychology. En P. A. Linley \& S. Joseph (Eds.), Positive psychology in practice. New York: John Wiley. 
Jacobson, N.S., \& Hollon, S.D. (1996). Cognitive-behavior therapy versus pharmacotherapy: Now that the jury's returned its verdict, it's time to present the rest of the evidence. Journal of Consulting and Clinical Psychology, 64, 74-80.

Jacobson, N.S. \& Truax, P. (1991). Clinical significance: A statistical approach to defining meaningful change in psychotherapy research. Jouyrnal of Consulting and Clinical Psychology, 59, 12-19.

Klein, D.F. (1996). Preventing hung juries about therapy studies. Journal of Consulting and Clinical Psycho$\log y, 64,81-87$.

Lambert, M.J. (1992). Implications of outcome research for psychotherapy integration. En J.C. Norcross \& M.R. Goldfried (Eds.), Hanbook of psychotherapy integration. New York: Basic Books.

Linley, P.A., \& Joseph, S. (Eds.) (2004). Positive psychology in practice. Hoboken, NJ: John Wiley \& Sons.

Lohr, J.M., Hooke, W., Gist, R., \& Tolin, D.F. (2003). Novel and controversial treatments for trauma-related stress disorders, En S.O. Lilienfeld, S.J. Lynn \& J. M. Lohr (Eds.), Science and pseudoscience in clinical psychology. New York: The Guilford Press.

Lopez, S.J., \& Snyder, C.R. (Eds.). Positive psychological assessment: A handbook of models and measures. Washington, DC: American Psychological Association.

Lopez, S.J., Snyder, C.R., Magyar-Moe, J.L., Edwards, L.E., Pedrotti, J.T., Janowski, K., Turner, J. L., \& Pressgrove, C. (2004). Strategies for accentuating hope. En P.A. Linley \& S. Joseph (Eds.), Positive psychology in practice. Hoboken, NJ: John Wiley \& Sons.

Lynn, S.J., Lock, T., Loftus, E. F., Krackow, E., \& Lilienfeld, S.O. (2003). The remembrance of things past: Problematic memory recovery techniques in psychotherapy. En S.O. Lilienfeld, S.J. Lynn \& J. M Lohr (Eds.), Science and pseudoscience in clinical psychology. New York: The Guilford Press.

Miller, D.S., Hubble, M.A., \& Duncan, B.L. (1996, march). Psychotherapy id dead, long live psychotherapy. Workshop at the $19^{\text {th }}$ Annual Family Therapy Network Symposium, Washington DC.

Nathan, P.E., \& Gorman, J.M. (1998). A guide to treatment that work. New York: Oxford University Press.

Ogles, B.M., Lambert, M.J., \& Sawyer, J.D. (1995). Clinical significance of the National Institute of Mental Health Treatment of Depression Collaborative Research Program dada. Journal of Consulting and Clinical Psychology, 63, 321-326.

Pelechano, V. (1973). Personalidad y parámetros. Tres escuelas y un modelo. Barcelona: Vicens Vives.

Pelechano, V. (1977). La evaluación de la psicoterapia. Valencia: Promolibro.
Pelechano, V. (1979). Psicología educativa comunitaria. Valencia: Promolibro,

Pelechano, V. (1980). Psicología familiar comunitaria. Valencia: Alfaplús.

Pelechano, V. (1989). Ejes de referencia y una propuesta temática. En E. Ibáñez y V. Pelechano (Eds.), Personalidad. Madrid: Alambra.

Pelechano, V. (2000). Psicología sistemática de la personalidad. Barcelona: Ariel.

Pelechano, V., y Botella, C. (1983). Apuntes de psicoterapia y modificación de conducta. Valencia: Promolibro, 1983.

Pelechano, V., y Servando, M. A. (2004). ¿Qué es la personalidad? Madrid: Biblioteca Nueva.

Rachman, S. (1979). The effects of psychotherapy. London: Academic.

Ramana, R., Paykel, E. E., Cooper, Z., Hayburst, Saxty, M., \& Surtees, P. G. (1995). Remission and relapse in major depression. Psychological Medicine, 25, 11611170.

Ruini, C., \& Fava, G.A. (2002). Well-being therapy of generalized anxiety disorder, Paper presented at the 2nd Positive Summer Institute, Philadelphia, August, 9-15.

Ruini, C., \& Fava, G.A. (2004). Clinical applications of well-being therapy, En P.A. Linley \& S. Joseph (Eds.). Positive psychology in practice, Hoboken, NJ, John Wiley \& Sons, pp.371-387.

Ryff, C.D., \& Singer, B.H. (1996). Psychological wellbeing: Meaning, measurement, and implications for psychotherapy research, Psychotherapy and Psychosomatics, 69, 170-177.

Seligman, M.E.P. (1993). What you can change and what you can't. New York: The Random House.

Seligman, M.E.P. (1995). The effectiveness of psychotherapy: The Consumer Report survey, American Psychologist, 50, 965-974.

Seligman, M.E.P. (1998). Afterword. En P. Nathan \& J. Gorman (Eds.), A guide to treatment that works. New York: Oxford University Press.

Singer, M.T., \& Nievod, A. (2003). New age therapies. En S.O. Lilienfeld, S.J. Lynn \& J.M. Lohr (Eds.). Science and pseudoscience in clinical psychology. New York: Guilford Press.

Sloane, R.B., Staples, F.R., Cristol, A.H., Yorkston, N.J., \& Whipple, K. (1975). Psychotherapy versus behaviour therapy. Cambridge, MA: Cambridge University Press.

Smith, M.L., \& Glass, G.V. (1977). Meta-analysis of psychotherapy outcome studies. American Psychologist, 132, 752-760.

Smith, M.L., Glass, G.V., \& Miller, T.L.(1980). The benefits of psychotherapy. Baltimore: Johns Hopkins University Press. 
Snyder, C.R. \& Lopez, S.J. (Eds.) (2005). Handbook of positive psychology. New York: Oxford University Press. Stricker, G. (1995). Failures in psychotherapy. Journal of Psychotherapy Integration, 5, 91-93.

Tedeschi, R.G., \& Calhoun, L.G. (2004). A clinical approach to posttraumatic growth. En P.A. Linley \& S. Joseph (Eds.), Positive psychology in practice. Hoboken, NJ: John Wiley \& Sons.
Wampold, B.E. (2001). The great psychotherapy debate: Models, methods and findings. Mahwah NJ: Erlbaum.

Wampold, B.E., Mondin, G.W., Moody, M., Stich, I., Benson, K., \& Ahn, H. (1997). A meta-analysis of outcome studies comparing bona fide psychotherapies: Empirically «all must have prizes». Psychological Bulletin, 122, 203-215. 